\title{
Features of Loschmidt's Number and Its Theoretical Frontiers
}

\author{
M.E. Zhussupov \\ Science, Noosphereorder Co., London, UK
}

Email: mjalmata@mail.ru

(Received July 2014; Published Sept 2014)

\begin{abstract}
Loschmidt's number has played an important role in the development of science and technology and in the establishment of various standards. However, despite advances in the technological methods of estimating Loschmidt's number, the nature of its formation remains unknown. The information that lies in the nature of this constant can be useful for understanding the properties of a physical vacuum and character of processes of the interaction of matter in this environment. Basing on time and experiment tested the laws of molecular physics and thermodynamics, some features of the host medium of micro-particles involved in the formation of Loschmidt's number were identified, describing its nature and the previously unknown phenomena of fundamental pressure of a Universe. The characteristics of various physical and chemical processes occurring in systems associated with a vacuum and the behavior of heavenly bodies are considered from the perspective of this phenomenon's existence.
\end{abstract}

Keywords: Loschmidt's number, fundamental pressure, gravitation, electromagnetism, physical vacuum

DOI:10.14331/ijfps.2014.330067

\section{INTRODUCTION}

Loschmidt's number is the number of micro-particles (atoms, molecules, electrons, or other similar objects) contained in one cubic meter of an ideal gas. The number of these particles (symbol $\mathrm{n}_{0}$ or $\mathrm{N}_{\mathrm{L}}$ ) in normal conditions (at a temperature of $273.15 \mathrm{~K}$ and a pressure of $101,325 \mathrm{~Pa}$ ) is numerically equal to $2.6867805 \times 10^{25}$ ("Loschmidt," n.d.). Avogadro (1811) discovered the law that "equal volumes of all gases at the same temperature and pressure have the same number of molecules," but the number of particles contained in these volumes became known only when the counting of gas molecules was made possible in 1865 by Johann Josef Loschmidt, whose method was based on the kinetic theory of gases that had been developed by Rudolf Clausius, Ludwig Boltzmann, and James Maxwell. Loschmidt, knowing the approximate size of molecules in air, as well as the length of their free path, measured the volume of liquid that condensed from a certain volume of gas and reported the first numerical evaluation of molecules. Following this, by 1932, approximately one hundred independent methods for experimentally determining this number had been developed by various researchers (Virgo, 1933). Many of these methods, including colloidal chemistry, $\mathrm{x}$-ray spectroscopy, and electronic and radioactive methods, had reached a substantial level of development, but the method based on electron charge evaluation was considered to be the most accurate. In this method, one measures the amount of electricity that is required for dissolving or precipitating 1 mole of silver. The ratio of this substance quantity to the electron charge, called the Faraday constant, gives Avogadro's number. One of the most recently measured values of the Andreas et al. (2011) was obtained during the research of a large group of scientists from Germany, Japan, Australia, Switzerland, the U.S., 
France, Italy, and Belgium (Andreas et al., 2011). The researchers worked on an almost ideally shaped sphere of silicon using laser interference, x-ray diffraction, and mass spectroscopy and were able to determine the atomic mass of silicon and the quantity of atoms in one mole of this substance with unprecedented accuracy. In practical terms, Loschmidt's and Avogadro's numbers have played important roles in the development of high-precision technologies, in the creation of the etalon of a kilogram, and in the simulation of physical-chemical processes, among others (Becker, 2012). These numbers are related to each other by the ratio given in $\mathrm{Eq}(1)$.

$$
N_{L}=\frac{N_{A}}{V_{M}}
$$

where $V_{M}$ is the molar volume (volume of one mole) of an ideal gas, which is equal to $0.022413996 \mathrm{~m}^{3}$ under normal conditions; and $N_{A}$ is Avogadro's number. Currently, using the CODATA-recommended values for physical constants (Mohr \& Taylor, 2005), Loschmidt's constant is calculated by $\mathrm{Eq}(2)$.

$$
n_{0}=\frac{N_{A} p_{0}}{R T_{0}}=\frac{A_{r}(e) M_{u} c \alpha^{2}}{2 R_{\infty} h R} \frac{p_{0}}{T_{0}}
$$

where $N_{A}$ is Avogadro's constant, $A_{r}(e)$ is the relative atomic mass of the electron, $M_{u}$ is the molar mass constant, $c$ is the speed of light, $\alpha$ is the fine-structure constant, $R$ is the gas constant, $R_{\infty}$ is the Rydberg constant, $h$ is Planck's constant, $p_{0}$ is the molecular pressure, and $T_{0}$ is the thermodynamic temperature. The precision with which Loschmidt's constant can be determined is limited only by uncertainty in the value of the gas constant. The aim this work is to identify causal relationships of forming Loschmidt's number with conditions of the physical vacuum which enclose the particles. An attempt to solve this problem is presented with the use of some of the laws of molecular physics and thermodynamics. This paper focuses on searching for interrelations among different physical quantities, which can then lead to logical findings regarding their nature.

\section{THE NATURE OF LOSCHMIDT'S NUMBER}

Despite the development of methods for quantifying Loschmidt's number, the nature of the formation of this number still remains unexplored. With the exception of molecular pressure and temperature, there is no information about the cause-and-effect relationship between the accumulation of particles in a given space and the physical and chemical conditions of the enclosing environment. A significant contribution to our knowledge of the nature of Loschmidt's number was made by Perrin (1923), who showed that Avogadro's law is valid not only for molecules and atoms but also for colloidal particles, which can be used for monitoring the quantification of values in an optical microscope. Furthermore, Perrin's experiments on the Brownian motion of colloidal particles served as important evidence for the atomic-molecular theory of the structure of matter (Perrin, 2013). His experiments showed that under standard conditions, all relatively thin, homogeneous particles tend to gravitate to the value of Loschmidt's number, independent of the type of substance. Since then, the study of the behavior of particles forming a system has grown into a separate branch of physics-statistical mechanics-the methods of which are based on concepts inherent in the microcosm and come from a cause-and-effect relationship between micro- and macro-phenomena. The microscopic and macroscopic versions of fluid and gas mechanics have one qualitative difference. Microscopic processes are characterized as reversible, while macroscopic processes are, in general, irreversible. However, as shown by recent studies (Hoover \& Hoover, 2003), macro and microstates have a probabilistic relationship that distinguishes the dependence of the macroscopic properties of the system on its microscopic properties. This work is based on empirical data and mathematical descriptions of phenomena that point to the continuity of processes occurring at the micro and macro levels. Accordingly, it follows that the irreversibility of macroscopic processes is a consequence of the growth of chaos (an increase in entropy) at the micro level, from which the particles are not able to return by themselves. This fact indicates the prevailing one-sided character of the manifestation of forces in the processes of particle transport in the considered environment, which has two components, vacuum and micro-particles. The physical vacuum that contains these particles remains the most probable source of these forces. Particles in the process of forming Loschmidt's constant seem, by all appearances, to occupy a passive position. The expected constancy of the number of particles in a certain amount of space reflects the structure and properties of the medium containing the particles.

For a long time, the concept of a vacuum was synonymous with complete emptiness, in which nothing happens and nothing can occur because there are no material particles or energy. However, with the development of quantum field theory (quantum electrodynamics), it was found that a vacuum can be viewed as an environment in which special virtual-i.e., "unobservable"-processes (Ryder, 1985) exist. The term "physical vacuum" was then introduced, which indicates the localization of virtual particles that are continuously produced for brief moments and then disappear. For example, researchers at the University of California experimentally confirmed the existence of the Casimir effect (Mohideen \& Roy, 1998), which is based on the mutual attraction of uncharged conductive bodies as a result of fluctuations of virtual particles in a physical vacuum. In this respect, the experiments at CERN on the Large Hadron Collider are of considerable interest to scientists because they have shown the possibility of synthesis from a physical vacuum of material particles (CERN, 2013). Thus, the vacuum has special physical properties that point to its materiality. To date, the properties of a physical vacuum postulated by quantum mechanics do not allow for an explanation of the regularities of the formation of Loschmidt's number. Adequate mathematical tools are not available for solving this problem. However, in the fields of molecular physics and thermodynamics, reliable and exact methods have been developed for studying the forces responsible for different states of thermodynamic systems. With respect to the mathematical tools, there is currently not 
enough statistical data to verify the correlation dependence between the studied phenomena and physical processes. From the fundamental equation of thermodynamics (3), it follows that in a gas phase, the work of motion and the interactions of molecules, atoms, electrons, and other particles depend on Gibbs energy and pressure, as follows,

$$
\Delta G_{T}=-R T \ln P=\Delta H_{T}-T \Delta S_{T}
$$

where $\Delta G_{T}$ is the change in Gibbs energy, $P$ is the pressure, $R$ is the ideal gas constant, $T$ is the temperature, $\Delta H_{T}$ is the change in enthalpy, and $\Delta S_{T}$ is the change in entropy. Enthalpy and entropy are parameters of the thermochemical characteristics of individual substances, while the Gibbs energy and pressure characterize the overall state of the environment. Because the thermodynamic parameter of temperature is associated with the physical vacuum via the Stefan-Boltzmann law (1879), it is logical to assume that a vacuum has its own pressure. From the standpoint of thermodynamics, one might hypothesize that in a certain volume of physical vacuum, in which there is only one electron to move, (i.e., to produce work), an influence of pressure is necessary. Here and below, electrons are conditionally presented in the form of spherical objects Fig (1). However, an actual electron is not localized in space as a separate particle. Rather, the electron is in constant motion in the form of streams and waves of different structural forms.

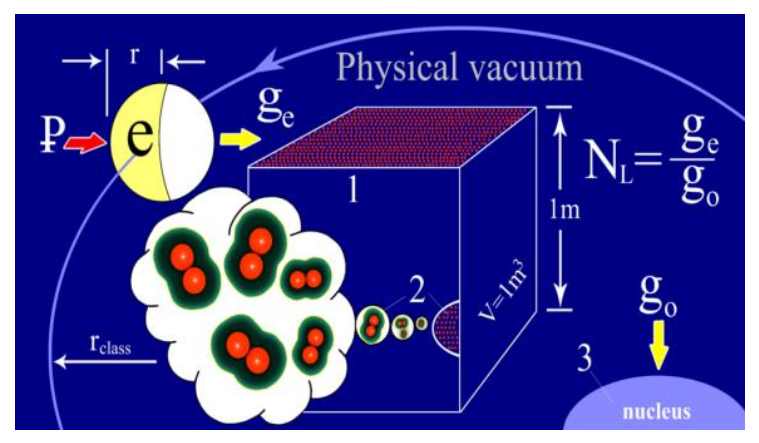

Fig 1. The scheme of formation of Loschmidt's number $\left(\mathrm{N}_{\mathrm{L}}\right)$ in the medium of a physical vacuum. $P$ is the fundamental pressure, $e$ is the electron, $r$ is the radius of the electron, $r_{\text {class }}$ is the classical radius of the electron, $g_{e}$ is the electron's acceleration due to pressure, $g_{0}$ is the acceleration of the electron in free fall, 1 is one cubic meter of space with gas, 2 is the molecules of oxygen in an electron shell, and 3 is the core of the atom.

Under the influence of such pressure, the electrons will experience acceleration with a force $(\mathrm{F} 1)$ equal to the product of the pressure and the area of the electron's surface with the classical radius, Eq (4). The classical electron radius, unlike its actual radius, is a fundamental constant and represents the radius of the circle exhibited by the electron in space. The force resulting from the physical vacuum's pressure on a single electron is as follows,

$$
F_{1}=P 2 \pi r^{2} \text { clas }
$$

where $P$ is the fundamental pressure and $r_{\text {clas }}$ is the classical radius of the electron. Electrons have a rest mass and thus can be influenced by the gravitational acceleration of any celestial body. The gravitational acceleration is directed in only one direction, while the fundamental pressure is static and operates in all directions, including the direction opposite to gravity. Objects experience a free-fall acceleration on the Earth at the mid-latitudes of $9.8 \mathrm{~m} / \mathrm{s}^{2}$, and coupled with the force of the fundamental pressure, we can use this to determine the existing value of the Loschmidt's number. The force of gravity $\left(F_{2}\right)$ is expressed in Eq (5) as follows:

$$
F_{2}=m_{e} g_{0}
$$

where $m_{e}$ is the mass of the electron and $\mathrm{g}_{0}$ is the free-fall acceleration. Thus far, it is necessary to consider only these two forces, while considering all of the other options as implicit. In this case, the filling of free space in vacuum will continue until one reaches the point at which the two forces applied to the electrons (gravity and pressure) are equal to each other. The force resulting from this pressure on the electron is much greater than the force of gravity and provides the localization of electrons in a minimal volume. Accordingly, the number of localized electrons is expressed in $\mathrm{Eq}(6)$

$$
N_{L}=\frac{P 2 \pi r^{2} \text { clas }}{m_{e} \mathrm{~g}_{0}}
$$

Loschmidt's number, as mentioned above, is empirically determined with high accuracy, and hence, there is no need to calculate it. Inasmuch as the value of the free-fall acceleration and other fundamental constants are already known, one can assess the value of the alleged pressure of a physical vacuum. The obtained value of the pressure of a physical vacuum was found to be $4.805 \times 10^{24} \mathrm{~Pa}$. Because the alleged pressure is manifested from the physical vacuum, which contains all observed objects, it is called the Fundamental Pressure of the Universe. This pressure is assumed to interact only with elementary particles. The manifestation of Loschmidt's number is explained by the mechanical interaction between the electrons in the structures of various particles and the medium of the physical vacuum. The number of particles that fill the space is controlled by the pressure of the physical vacuum acting on the total surface of the electrons. The free electrons, similar to molecular and atomic particles, will most likely uniformly fill the free space equivalent to the Loschmidt number under a gravitational acceleration of $9.8 \mathrm{~m} / \mathrm{s}^{2}$.

\section{THE FUNDAMENTAL PRESSURE OF THE UNIVERSE}

The fundamental pressure exerted on the surface of electrons in atoms and molecules provides a counteraction to the force of gravity and their uniform distribution in space. The balance of these forces is achieved in normal conditions with amounts of various micro-particles equal to Loschmidt's number. It is relatively acceptable to insert the parameter of this pressure into the equations from a thermodynamics position, but an adequate explanation is required from the standpoint of theoretical physics. As is already known, the unification of the four fundamental interactions in physicsgravity, electromagnetism, and the weak and strong nuclear 
forces-remains unsolved. Historically, ideas about these interactions have consistently been put forth, and researchers have studied the phenomena related to these interactions. Simultaneously, for the purpose of creating a complete and objective picture of physical laws, research has been conducted on the ways in which these phenomena are associated-for example, such an experiment was successfully executed at the end of the XIX century for electromagnetism. Presently, the possibility of combining electromagnetism and the weak fundamental force is being actively discussed. Existing theories of interactions-e.g., the quantum theory of electrodynamics and quantum chromodynamics-are now only considered as low-energy approximations of the framework of this unification. Obviously, the solution to this problem depends on the diversity and reliability of the methods for determining the sources of strength and energy of various physical processes. In this work, pressure is represented as the most probable source of force for all four fundamental interactions. This conclusion is based on the elaboration of thermodynamic and astrophysical criteria for association among the four fundamental interactions (M. Zhussupov, 2011a,; M. Zhussupov, 2011b). In Eq (1) above, Gibbs energy is the work of a thermodynamic system against exterior forces, such as the force of pressure. However, at low pressures, the applicability of this equation is severely limited, and it does not work at all in a vacuum because a parameter of the gas pressure is used in this equation. As is known, most components of thermodynamic systems in nature-e.g., planets, moons, comets, and asteroids-are permanently in a vacuum and are frequently exposed to hightemperature conditions. At the same time, these components are in physical and chemical equilibrium with the environment. For example, it is known that the surface of the planet nearest to the sun, Mercury, heats up in the daytime to $720 \mathrm{~K}$, at an atmospheric pressure of less than $2 \times 10^{-7} \mathrm{~Pa}$. Xray and gamma-ray spectrometers on NASA's Messenger spacecraft are obtaining key data about the composition and properties of the surface of Mercury (Martel, 2013). This work shows that the lava on Mercury is most similar to terrestrial magnesium basalt, primarily consisting of oxides of silicon, potassium, sodium, magnesium, and aluminum. In accordance with the equation of temperature dependence of saturated steam pressure in similar conditions, more than half of the oxides in the composition of these rocks must rapidly evaporate. However, judging by the magnitude of the atmospheric pressure of this planet, this expectation does not occur in reality. Considering that the fundamental equation of thermodynamics is applicable to any thermodynamic system, it is possible to assume that in the vacuum of the planet Mercury, there is an unknown pressure that promotes the occurrence of Gibbs energy. The force of this pressure in the condition of a physical vacuum can limit the evaporation of the oxides in the absence of molecular pressure. For thermodynamic systems in a vacuum condition, the involvement of fundamental pressure can be expressed by $\mathrm{Eq}(7)$, as follows,

$$
P=\frac{D\left(\mathrm{~g}_{0}+\mathrm{g}_{T}\right)}{A}
$$

where $D$ is the density of the system, $\mathrm{g}_{0}$ is the free-fall acceleration, $g_{T}$ is the kinetic acceleration of the molecules, and $A$ is the surface area of the molecules. The above equation reflects the state of the chemicals irrespective of the atmospheric pressure. This report provides the first attempt to use the parameter of fundamental pressure for thermodynamic systems in vacuum. The parameter of fundamental pressure has been used in thermodynamics equations in the structure of an algorithm for ab initio (first principles) computer simulations of physical and chemical processes and properties of minerals (M. Zhussupov, 2011c, ). As is already known, gravitation has become a cornerstone of modern science but is unamenable to most physical models. Astrophysical criteria for the association of the four fundamental interactions indicate the existence of an alternative to the force of gravity. The manifestation of wave motion expressed in the periodicity and constancy of trajectories is characteristic of celestial bodies. Additionally, in accord with Newton's first law, the characteristic motion of heavenly bodies remains unchanged without the involvement of external forces. De Broglie (1923) regarding wave-particle dualism in nature is applicable to any material body, but his formula (Eq 8) works only for describing the wave motion of elementary particles.

$$
\lambda=\frac{h}{m v}
$$

where $\lambda$ is de Broglie's wavelength, $h$ is Planck's constant, $m$ is the rest mass of an electron, and $v$ is the velocity of the electron. It is believed that for macroscopic objects, this equation is unacceptable because it gives a negligible value for the wavelength. Contrary to this view, it can be shown that under the influence of fundamental pressure, the de Broglie equation may possess additive properties. In other words, the wavelength of a macro object increases proportionally to the quantity of structural units of space commensurate with de Broglie's electron wave contained in its volume. Energetically equivalent to one such structural unit of space, the electron wave is equal to the ratio of electron mass to the magnetic permeability divided by $\pi$. For the Earth, the permeability is equal to the magnetic constant. The total number of structural units of space is equal to the ratio of the product of fundamental pressure and the volume of the torus produced by the object to the energetic equivalent of one such structural unit, as shown in Eq (9)

$$
n=\frac{P V \mu}{\pi m_{e}}
$$

where $V$ is the volume of the torus produced by the object, $m_{e}$ is the electron mass and $\mu$ is the magnetic permeability. Inputting the resulting number into the numerator of de Broglie's equation enables us to calculate the true wavelength of macroscopic objects, as shown in Eq (10)

$$
\lambda=\frac{h n}{m v}=\frac{h}{m v} \frac{P V \mu}{\pi m_{e}}
$$

where $\mathrm{m}$ is the mass of the Earth and $v$ is the speed of the Earth moving around the sun $(29,780 \mathrm{~m} / \mathrm{s})$. As an example, consider the nature of the wave motion of the Earth relative to the Sun (Fig. 2). 


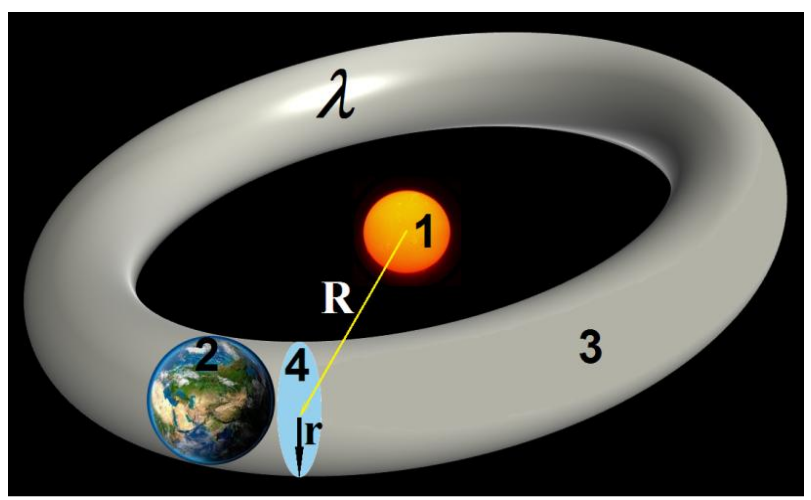

Fig 2. A non-gravitational scheme of wave movement of the Earth round the Sun. 1 is the Sun, 2 is the Earth, 3 is the torus produced by the geosphere of the Earth, 4 is a cross section of the
Earth and of the torus, $r$ is the radius of the Earth, $\mathrm{R}$ is the radius of the Earth's orbit or the amplitude of its wave, and $\lambda$ is the de Broglie wavelength for the Earth.

The volume of the torus produced by the geosphere of Earth is equal to the product of its cross section $\left(\pi r^{2}\right)$ and the length of the orbit around the Sun $(2 \pi R)$. Substituting the obtained value of the torus volume into $\mathrm{Eq}(10)$ gives a de Broglie wavelength of 941.6 million kilometers, which almost exactly coincides with the length of the orbit of the Earth around the sun. This equation was also verified by calculating the parameters of the other planets of the solar system (Table 1).

Table 1. Calculated and tabulated parameters of the solar system planets. $\mathrm{K}$ is the ratio of the calculated and tabulated data; $\mu$ is the calculated magnetic permeability of the planets. Source of tabulated data: NASA.

\begin{tabular}{|c|c|c|c|c|c|c|}
\hline \multirow{2}{*}{ Name of Planet } & \multicolumn{2}{|c|}{$\underline{\text { Orbital Radius (m) }}$} & \multicolumn{2}{|c|}{ Mass of Planet (kg) } & \multirow{2}{*}{$\mathbf{K}$} & \multirow{2}{*}{ Permeability $(\mu)$} \\
\hline & Calculated & Tabulated & Calculated & Tabulated & & \\
\hline Mercury & $9.4806 \times 10^{10}$ & $5.79092 \times 10^{10}$ & $5.4182 \times 10^{23}$ & $3.3022 \times 10^{23}$ & 1.64 & $7.6900 \times 10^{-7}$ \\
\hline Venus & $1.0219 \times 10^{11}$ & $1.08209 \times 10^{11}$ & $4.6252 \times 10^{24}$ & $4.8685 \times 10^{24}$ & 0.95 & $1.3283 \times 10^{-6}$ \\
\hline Earth & $1.4922 \times 10^{11}$ & $1.49598 \times 10^{11}$ & $5.9659 \times 10^{24}$ & $5.9736 \times 10^{24}$ & 1.00 & $1.2636 \times 10^{-6}$ \\
\hline Mars & $7.3737 \times 10^{11}$ & $2.27943 \times 10^{11}$ & $2.0839 \times 10^{24}$ & $6.4185 \times 10^{23}$ & 3.25 & $3.8867 \times 10^{-7}$ \\
\hline Jupiter & $6.6903 \times 10^{11}$ & $7.78340 \times 10^{11}$ & $1.6391 \times 10^{27}$ & $1.8986 \times 10^{27}$ & 0.87 & $1.4617 \times 10^{-6}$ \\
\hline Saturn & $3.8507 \times 10^{12}$ & $1.42666 \times 10^{12}$ & $1.5389 \times 10^{27}$ & $5.6846 \times 10^{26}$ & 2.71 & $4.6614 \times 10^{-7}$ \\
\hline Uranus & $1.3636 \times 10^{13}$ & $2.87065 \times 10^{12}$ & $4.1396 \times 10^{26}$ & $8.6810 \times 10^{25}$ & 4.77 & $2.6473 \times 10^{-7}$ \\
\hline Neptune & $2.1370 \times 10^{13}$ & $4.49839 \times 10^{12}$ & $4.8857 \times 10^{26}$ & $1.0243 \times 10^{25}$ & 4.77 & $2.6457 \times 10^{-7}$ \\
\hline
\end{tabular}

In $\mathrm{Eq}(10)$ above, one variable parameter, on which the conformity between the calculated and tabulated data of the orbital radii is dependent, is the magnetic permeability. In detail, the Earth's magnetic field was studied as an analogue of a permanent magnet with poles located near the geographic poles. The environment, in which the magnetic field is manifested, is related to the value of magnetic permeability.

The average value of the absolute permeability of all earth materials is approximately $1.26 \times 10^{-6} \mathrm{H} / \mathrm{m}$. The calculated absolute permeability values of the other planets in the solar system are shown in Table 1 . These values are calculated according to $\mathrm{Eq}(10)$ and exhibit differences from the Earth's magnetic permeability. Due to the lack of data regarding the permeability of these planets in the literature, a qualitative assessment was carried out on based on information of the magnetic fields of these objects.

Closest to the Sun, the planet Mercury has a weak magnetic moment, approximately $4 \times 10^{-4}$ times that of the Earth. As a result of the low magnetic moment, the solar wind deflects the magnetic field at a distance of only 1.5 Mercury radii from the center of the planet (C. T. Russell, \& Luhmann, J. $\mathrm{G}, 1997$ ). For comparison, most of the solar wind around the Earth is deflected by the magnetic field at a distance of approximately 10 times the radius from the Earth's center. This fact may indicate a small value of the planetary magnetic permeability of Mercury, in accordance with its calculated value. Information about the magnetic field of the next planet in the solar system-Venus is very contradictory. Previously, it was thought that its magnetic field was much weaker than the Earth's field (C. T. Russell, 1981). Recently, however, astrophysicists have found that the magnetic field of Venus is more active than previously thought. An unusual magnetic phenomenon created giant magnetic bubbles around Venus. At present, Venus Express, a spacecraft of the European Space Agency, is in orbit around this planet. The results of magneto-metric measurements show that the planet does not generate magnetic fields within its interior; the plasma dynamics of Venus are very similar to those of Earth (Zhang et al., 2012).

Today, the magnetic field of Venus is believed to derive from the interaction between solar winds and the upper atmosphere. Specifically, the calculated value of the magnetic permeability of the planet, which is similar to the Earth's magnetic permeability, indicates that Venus's rocks may be magnetized. Mars has a weak global magnetic field with a strength $1.25 \times 10^{-3}$ of the Earth's magnetic field. In addition, Mars has a large spectrum of magnetic fields, which are often very strong but are manifested locally on a small scale.

Their occurrence may be associated with the spatial variation of remnant magnetism in the crust. Martian surface processes may be of great importance in the distribution of magnetic fields; research regarding this possibility will be conducted in the near future (Chassefière et al., 2004). The estimated value of the Martian magnetic permeability is also extremely low, which may be due to the total decrease in the 
magnetic susceptibility of rocks by the process of oxidation. Table 1 shows that Jupiter has the highest magnetic constant. Indeed, Jupiter has a large and intense magnetic field at the tops of its clouds, which is 20 times stronger than that of the Earth (NASA., 2011).

The calculated values of the magnetic permeability of the remaining objects in Table 1 are less than Earth's values, which is also in accordance with reported data on the forces of their magnetic fields. For example, the magnetic field strengths at the equator, at the top of the clouds of Saturn and at the surface of Uranus are less than the average value of the magnetic field on the surface of the Earth by a factor of approximately two.

Neptune's magnetic field strength is described as complex and non-constant and varies from a maximum of more than 1 Gauss in the southern hemisphere to a minimum of less than 0.1 Gauss in the northern hemisphere (NASA., 2014). Thus, a qualitative agreement is observed between the calculated permeability of the planets in the solar system with information about their magnetism from literature sources. The criteria listed above support the proposal that the vacuum represents a material medium, which manifests itself through force actions on observed physical objects.

The most likely universal source of this force is the fundamental pressure on elementary particles. The force resulting from this pressure could be the basis for the manifestation of the various gravitational, electromagnetic, strong and weak interactions.

\section{NUMERICAL VALUE OF THE FUNDAMENTAL PRESSURE}

A more accurate value can be obtained for the fundamental pressure than for Loschmidt's number by interpreting a known phenomenon of nuclear physics-neutron decay. It is known that while bound neutrons in nuclei are stable, a free neutron, which is released during a nuclear reaction, is highly unstable and decays into a proton and an electron. Part of the energy that is lost is usually attributed to the formation of the so-called electron anti-neutrino.

The decay of a neutron into a bound state occurs with the formation of a hydrogen atom or an electron-proton pair, as shown in Eq (11).

$$
n \rightarrow{ }_{1}^{1} P+e^{-} \rightarrow{ }^{1} H
$$

where $n$ is the neutron, $p$ is the proton, e is the electron, and $H$ is hydrogen. In this process, the stretching of the time of neutron decay is of interest, but its cause is still unclear. Free neutrons undergo beta decay, in which a mean lifetime under normal conditions is $893 \mathrm{~s}$ (Galanin, 1990).

These calculations indicate that the limit on the rate of release of an electron to the orbit imposes a fundamental pressure. Schematically, the decay of the neutron with the gradual removal of a newborn electron from the nucleus on a spiral to the orbit is shown in Fig (3).

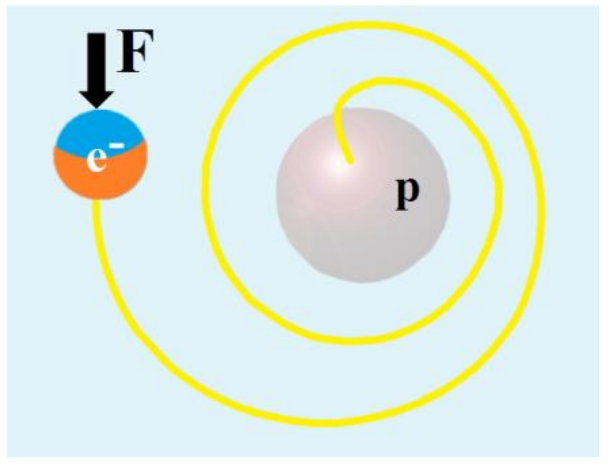

Fig 3. Schematic of the birth of an electron-proton pair from a neutron (weak fundamental interaction). $F$ is the force of fundamental pressure, $\mathrm{e}^{-}$is the electron, and $\mathrm{p}$ is the proton.

In this case, the fundamental pressure must be equal to the ratio of the work function of the electron as it travels to its orbit to the volume of the hydrogen atoms within the limits of the first Bohr orbit radius, which is known to be equal to $5.29 \times 10^{-10} \mathrm{~m} . \operatorname{Eq}(12)$.

$$
P=\frac{3 W_{e}}{4 \pi R^{3}}
$$

where $W_{e}$ is the work function of the electron traveling to its orbit and $R$ is the radius of the first orbit in a hydrogen atom.

The fundamental pressure is otherwise expressed by the ratio $\mathrm{Eq}(13)$ of the resistance force against an electron and the area of the electron's forward hemisphere in the direction of its movement (in the picture, this parameter is shown in blue).

$$
P=\frac{3 F}{2 \pi r^{2}}
$$

where $F$ is the resistance force against an electron and $\mathrm{r}$ is the electron radius. The volume of space occupied by an electron in the process of reaching its orbit $\left(V_{e}=l 2 \pi r^{2} / 3^{2}\right)$ is equal to the volume of the hydrogen atom within the limits of Bohr's orbit $\left(V_{a}=4 \pi R^{3} / 3\right)$. This volume is found from the product of the cross section of the electron and the distance traveled by the electron during the lifetime of the neutron. Hence, the electron radius is given by $\mathrm{Eq}$ (14), as follows,

$$
r=\sqrt{\frac{6 R^{3}}{l}}
$$

where $\mathrm{R}$ is the radius of the first orbit in a hydrogen atom and $l$ is the distance traveled by an electron, which is equal to the product of the speed and lifetime of the electron. The distance traveled by an electron is the ratio of the square of the electron velocity in the first Bohr orbit $(2,187,690 \mathrm{~m} / \mathrm{s})$ to its acceleration. This acceleration, corresponding to a uniformly accelerated motion, is equal to the ratio of the final electron velocity to the lifetime of the neutron. As a result, the electron radius was found to be equal to $2.136 \times 10^{-20} \mathrm{~m}$ (the corresponding value of the electron radius below is shown from the perspective of kinetic-molecular theory). The work function of an electron in orbit $\left(W_{e}\right)$ is expressed by the 
ratio of the product of Planck's constant and the speed of light to the electron radius, as shown in Eq (15).

$$
W_{e}=\frac{h c}{\pi r}
$$

where $h$ is Planck's constant, c is the speed of light, and $r$ is the electron radius. From the first two relationships for pressure, the force resistant to the movement of the electron is found. The final formula for the participation of the physical vacuum pressure in the process of neutron decay has the form found in Eq (16).

$$
P=\frac{3 h c}{4 \pi r \pi R^{3}}
$$

A value for the pressure of a physical vacuum or the fundamental pressure $\left(4.775 \times 10^{24} \mathrm{~Pa}\right)$ is obtained from this ratio, and this value, which depends only on the accuracy of the time measurement of neutron decay, is represented as more accurate than the previous value obtained from Loschmidt's number.

An alternate calculation of the radius of the electron may also be used. By analogy with the methods used to determine the size and mean free path of molecules in molecular physics, the value of the electron radius can be confirmed by the following calculation. For this it is necessary to agree that instead of atmospheric pressure, the alleged pressure of the physical vacuum will be considered, and instead of molecules, electrons will be considered. A certain volume of space discharges an electron into movement.

The product of this volume and the pressure of the physical vacuum must be equal to the product of the number of particles, the Boltzmann constant and the temperature i.e., $n k T$. This value is numerically equal to the molecular atmospheric pressure if the number of particles is considered to be equal to Loschmidt's number. The mean free path of electrons in a region of molecules and atoms numerically corresponds to the mean free path of these particles. The subject for the auxiliary calculation is a hydrogen atom, which has only one electron. In accordance with the kinetic theory of gases (Kuchling, 1980), the mean free path of gas molecules depends on the molecular pressure, the temperature, and the diameter of the molecule (Eq 17).

$$
l=\frac{k T}{\pi \sqrt{2} d^{2} p}
$$

where $k$ is the Boltzmann constant, $T$ is the temperature $(273.15 \mathrm{~K}), d$ is the molecule's diameter, and $p$ is the molecular pressure $(101,325 \mathrm{~Pa})$. The diameter of a hydrogen atom taken from the radius of its first Bohr orbit is equal to $1.058 \times 10^{-10} \mathrm{~m}$. Inputting these data into the formula gives a mean free path of $7.48 \times 10^{-7} \mathrm{~m}$ for the hydrogen atom. Taking this value as the mean free path of an electron can aid in the evaluation of its radius. The total volume of electrons within the limits of one cubic meter of space can be found in relation to the atmospheric pressure, the fundamental pressure and the number of electrons, which is equal to Loschmidt's number. Accordingly, from the numerical ratio $101,325 / 4.775 \times 10^{24} / 2.68678 \times 10^{25}$, the volume occupied by a single electron can be computed, which turns out to be equal to $7.8978 \times 10^{-46} \mathrm{~m} 3$. This amount divided by the mean free path of an electron gives the cross-sectional area of the electron. Its numerical value $\left(1.833 \times 10^{-20} \mathrm{~m}\right)$ approximates the radius of the electron, which was obtained from an example of neutron decay. The value of the electron radius above, which was used to calculate the fundamental pressure, seems quite accurate. The probability of the existence of the electron radius should also be evident from the relationships among the fundamental constants. As is already known, the electron cloud of an atom of hydrogen with a charge e = $1.602176487 \times 10^{-19}$ contains $Q=1 / \mathrm{e}=6.241509647 \times 10^{18}$ electrons. The area of this cloud divided by the quantity of electrons gives the area of one electron; using this relation, $\mathrm{Eq}(18)$ gives the electron's radius.

$$
r=\sqrt{\frac{R^{2}}{Q}}
$$

In this case, the value is equal to $2.118147 \times 10^{-20} \mathrm{~m}$. This value is slightly smaller than the radii measured before, which may be the result of some overlap of the electron surface with other electrons. The calculated value of the electron radius is very similar to the ratio of the classical electron radius, the speed of light, and the classical electron speed of the first Bohr orbit, as shown in Eq (19)

$$
r=\frac{r_{\text {clas }} v}{\pi^{6} c}
$$

where $r_{\text {clas }}$ is the classical electron radius, $v$ is the electron speed of the first Bohr orbit, and $c$ is the speed of light. Substitution of the numerical values gives a radius value essentially equal to the calculated value $-2.138933 \times 10^{-20} \mathrm{~m}$. Therefore, the electron acquires a real shape, and its radius is approximated at $2.136 \times 10^{-20} \mathrm{~m}$. The process of neutron decay is presented as an example of a weak fundamental interaction. Similar calculations were also carried out for the two remaining fundamental interactions-the electromagnetic and strong (nuclear) interactions (M . Zhussupov, 2013).

In these calculations, the possibility of unifying the fundamental interactions is shown based on the corresponding interpretation of Ampère's force law and the structural features of the helium-4 nucleus. Ampere's law of 1820 is one of the classic examples of electromagnetic interaction and is based on the nature of the mechanical interactions of conductors carrying direct current. According to this law, parallel conductors with constant currents flowing in the same direction attract each other, and in the case of currents flowing in opposite directions, the conductors repel each other (Fig. 4a).

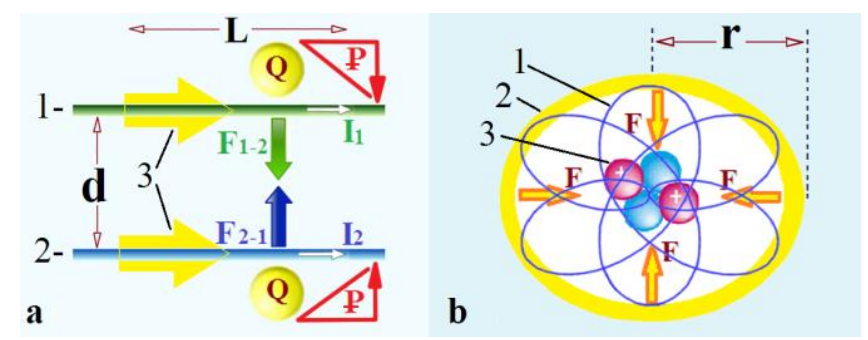

Fig.4 Schematic diagram of manifestations of the interactions of the electromagnetic and strong nuclear forces: (a) $\mathrm{L}$ is the length of 
parallel conductors with currents $\mathrm{I}_{1}$, and $\mathrm{I}_{2}, \mathrm{~d}$ is the distance between conductors 1 and 2, 3 shows the direction of motion of the electrons, and $\mathrm{P}$ Q shows the total counteraction of the pressure and electrons with the formation of the resultant forces $F_{1,2}$ and $F_{2,1}$. (b) $F$ is the vector direction of the fundamental pressure, $\mathrm{r}$ is the core radius of helium- 4 in a lepton's shell, 1 is the lepton wave, 2 is the lepton's shell, and 3 represents the nucleons.

It was experimentally established by Ampere that, in a vacuum, the force per meter of length between two parallel conductors spaced $1 \mathrm{~m}$ apart, with each carrying a current of $1 \mathrm{~A}$, is equal to $2 \times 10^{-7} \mathrm{~N}$. Regarding the mechanism of interaction between the conductors, complicated concepts exist based on quantum field theory. Without describing this quantum mechanical representation, it is possible to ensure that the mechanism of the behavior of conductors under the action of fundamental pressure appears simpler. Applied to electromagnetic interactions in the creation of mechanical tension in a medium containing electrons, the participation of this pressure is manifested. The force of this pressure increases in proportion to the area of the electrons, which experience frontal resistance and lose their impulse. This impulse is transferred to the environment and creates therein a field of tension-the analog of an electromagnetic field. The product of the effective volume of this field and the fundamental pressure, taking the fine-structure constant into consideration, represents the work that is responsible for the behavior of the conductors. The effective volume is the pure volume of the electrons, which can be calculated based on the classical electron radius with Eq (20) below.

$$
V_{e}=Q \frac{4 \pi r^{3} \text { clas }}{3}
$$

where $Q$ is the number of electrons in the current with a force of $1 \mathrm{~A}$ and $r_{\text {clas }}$ is the classical electron radius. The action of the force in the interaction of conductors occurs in one plane; therefore, one-third of the total pressure is applied. In the calculations, it is necessary to take into consideration the configuration of an electromagnetic field that is created around a conductor by the electrons moving in it. For a field source segment of conductor with length $\mathrm{L}$, the radius of the section of this field is assumed to be equal to $L / 2 \pi$. The ratio of this radius to the distance between the conductors (in this case, $1 \mathrm{~m}$ ) in bulk proportions will most likely be directly proportional to the force of their interaction. Thus, the work of fundamental pressure for current-carrying conductors takes into account the fine-structure constant and is expressed by $\mathrm{Eq}(21)$ as follows,

$$
W=\frac{P V_{e}}{3}\left(\frac{L}{2 \pi d}\right)^{3} \alpha^{2}
$$

where $\alpha$ is the fine-structure constant (constant of electromagnetic interaction). The resulting value of this work corresponds exactly to the moment of force of the Coulomb interaction between current-carrying conductors in an interpreted version of Ampere's law. To explain the mechanism of behavior of the conductors, one can use an analogy with Daniel Bernoulli's law (1738). Supposedly, in the physical vacuum between conductors, the movement of virtual particles in one direction occurs at the expense of sweeping out by electrons. Due to this phenomenon, the pressure decreases in the field, which can attract the conductors. When the motion of electrons in the conductors occurs in opposite directions, counter flows will increase the pressure and will push the conductors apart.

As is already known, the force of the strong interaction exceeds the forces of other fundamental interactionselectromagnetic, weak, and gravitational by a great extent. This force arises at a relatively short distance, which is commensurate with the size of the atomic nucleus, and is responsible for communication among nucleons (protons and neutrons).

According to modern representations, this interaction accounts for constant exchange between the nucleons of hypothetical particles-gluons-which seek, as though glued together, the quarks in their composition. However, this theory still requires a logical conclusion, which currently depends on the technical problems related to experimental studies. Presumably, the involvement of fundamental pressure in strong interactions could greatly simplify the binding mechanism of the nucleons. In particular, this approach frees the nucleons from the initiative of forming bonds with each other. A large numerical value for fundamental pressure could serve as a guarantee for the existence of a strong interaction. Being clamped inside an electronic (lepton) shell by the force of this pressure, the nucleons are compelled to interact, submitting to the symmetry of the environment. The compression force of the atomic nucleus in this case is directly proportional to the area of its electron shell. It is probable that a generic relationship of fundamental pressure with a strong interaction can be calculated based on the energy balance of the nucleus of helium-4, which consists of two protons and two neutrons (Fig. 4b). The binding energy of the core of an atom per nucleon is equal to the product of the mass defect and the square of the speed of light divided by the total number of nucleons. For the helium nucleus, this energy is $7.074 \mathrm{MeV}$. The nucleons are relatively small, and the effect of the pressure force on their surfaces would be considerably less than their binding force.

However, it is necessary to take into consideration the fact that the existence of separate nucleons in the form of naked cores is impossible. Protons are necessarily associated with electrons, and a neutron in the free form immediately decays and turns into a proton. Therefore, it is necessary to determine the minimum radius of the nucleon in the lepton's "shell", in which the nucleon can most likely participate in the processes of thermonuclear synthesis before merging with other nucleons. Obviously, this radius should not be less than the reduced Compton wavelength of the electron because the electron cannot be considered a real object otherwise. Simple multiplication of the fundamental pressure and the volume of a sphere with a radius equal to the reduced Compton wavelength gives an energy value equal to $7.189 \mathrm{MeV}$ per nucleon. This exact amount of energy is released in neutron deuteron nuclear reactions, according to data provided by the Nuclear Energy Information Center of Japan (JENDL-4.0) 
(Nuclear Data Center). Equation (22), which contains the parameter of fundamental pressure, the de Broglie wavelength, and other fundamental constants, yields a more approximate value of the binding energy of the helium-4 nucleus.

$$
E=\frac{M m}{\lambda} \frac{c^{2} \omega^{2}}{P}
$$

where $M$ is the mass of the nucleon, $m$ is the mass of the electron, $c$ is the speed of light, $\lambda$ is the reduced Compton wavelength, and $\omega$ is the frequency of the field, which equals $c / \lambda$, where $\lambda$ is the Compton wavelength. This equation can be equally applied to the gravitational interaction because its far right-hand term gives the value of the gravitational constants at the respective frequencies of the field, as expected from the law of universal gravitation and strong gravitation, as in Salam's version (Salam \& Sivaram, 1993). Specified criteria for the association of fundamental interactions are developed upon a common principle of the mutual relations of separate electrons and of parts of different objects (atoms, molecules, crystals, etc.) with forces of the fundamental pressure of the universe. For potential objects of nature that interact with fundamental pressure, this paper considered only electrons. The nucleon surface in the nucleus is most likely too small to create a significant force for the direct participation of fundamental pressure in inter-nuclear processes. In contrast, the surfaces of atoms are too large for the stress of the fundamental pressure. For example, with the assumption that the surface of the hydrogen atom fully reflects the burden of the fundamental pressure within the limit of the first Bohr orbit, the value could be estimated as 18.5 TeV. Therefore, all of the arguments in this paper are based on the assumption that the surface of an object larger than the electron, such as an atom, molecule, crystal, etc., is transparent to the fundamental pressure. The modern technologies for measuring pressure are configured based on the perception of pressure on observed material objects (e.g., molecules, atoms, electrons, photons), and thus, they are currently unable to trace fundamental pressure. In any case, fundamental pressure may prove to be a creative force responsible for the interatomic and intermolecular interactions of all of the objects of nature, from heavenly bodies to living organisms.

\section{CONCLUSION}

The physical vacuum as a real form of matter on various scales of nature is rather difficult to investigate. Nonetheless, to date, its existence has been demonstrated, and experiments have been conducted to identify its composition and properties. However, such studies of this issue, aside from apparent theoretical contradictions, are delayed due to some unresolved technical problems. In addition to empirical methods, which rely on observation and experimentation of the medium itself, significant information about the physical vacuum can be derived by studying the types, forms and properties of substances contained within. This paper focuses, therefore, on the problems associated with the genetic features of Loschmidt's number of a substance, drawing attention to the lack of research in the existing literature in this direction. It has been shown that this deficiency has led to a corresponding shortage of information regarding the interpretation of properties of vacuum media containing elementary particles. Loschmidt's number carries genetic information of interest for theoretically interpreting the nature of a physical vacuum. The alleged pressure of a physical vacuum follows as a consequence of the law of molecular physics and the equations of thermodynamics. The nature of this number is represented as a result of the mechanical interaction of electrons (moving freely or as a part of various particles) under the influence of this medium. The emergence of Loschmidt's number arises from the drive toward the mutual equilibrium of two forces-the pressure of the physical vacuum on the surface of the electrons and the force of the free-fall acceleration of the particles that contain these electrons. This pressure exists independently of the molecular pressure (atmospheric or mining) and arises from the physical vacuum of space, which contains in itself all of the observed objects, from elementary particles to galaxies. For this reason, this parameter has been named the Fundamental Pressure of the Universe. The force of this pressure is responsible for the occurrence of all four fundamental interactions (gravitation, electromagnetism, and strong and weak interactions) and indicates their single mechanical principle of manifestation. First, all tangible objects, including organic cells, can exist in and shape its structure depending on the nature of the manifestation of the fundamental pressure and the corresponding behavior of electrons. The fundamental pressure is more than $4 \times 10^{19}$ times greater than atmospheric pressure. However, in technical terms, it must be considered that the surfaces of atoms, molecules, crystals, etc., which are not occupied by electrons, are transparent to this fundamental pressure. The numerical value of this pressure is determined on the basis of Loschmidt's number and is confirmed by interpreting neutron decay data. In this research, cause-and-effect relationships of phenomena are identified as physical, as opposed to mathematical. A lack of mathematical means for describing results prevents one from creating a sufficiently coherent picture of interrelations of physical magnitudes and phenomena. However, in combination with Loschmidt's number and other constants, a parameter of the fundamental pressure can be introduced into the equations of thermodynamics to promote their application to systems associated with vacuum processes, particularly for astrophysical objects. In addition, the features of gravitation, electromagnetism and nuclear and particle physics are employed to expand the frontiers of knowledge regarding the structure and other vital properties of outer space.

\section{ACKNOWLEDGMENTS}

This work was carried out with the support of the organizers of the Thermodynamics Conference 2011, particularly Chairman Professor Ioannis Economou, and the Thermodynamics Conference 2013, particularly Chairman Professor Andrew Masters. I would like to express my deepest gratitude to the participants at these significant events for their encouragement and comments. 


\section{REFERENCES}

Andreas, B., Azuma, Y., Bartl, G., Becker, P., Bettin, H., Borys, M., Fujii, K. (2011). Determination of the Avogadro Constant by Counting the Atoms in a Si 28 Crystal. Physical review letters, 106(3), 030801.

Avogadro, A. (1811). Essai d'une manière de déterminer les masses relatives des molécules élémentaires des corps, et les proportions selon lesquelles elles entrent dans les combinaisons.

Becker, P. (2012). The new kilogram definition based on counting the atoms in a $28 \mathrm{Si}$ crystal. Contemporary Physics, 53(6), 461-479.

CERN. (2013). New Results Indicate that New Particle Is a HiggsBoson.http://home.web.cern.ch/about/updates/2013/0 3/new-results-indicate-new-particle-higgs-boson

Chassefière, E., Bertaux, J.-L., Berthelier, J.-J., Cabane, M., Ciarletti, V., Durry, G., Menvielle, M. (2004). MEP (Mars Environment Package): toward a package for studying environmental conditions at the surface of Mars from future lander/rover missions. Advances in Space Research, 34(8), 1702-1709.

De Broglie, L. (1923). Waves and quanta. Nature, 112(2815), 540-540.

Galanin, A. (1990). Introduction to the Theory of Nuclear Reactors Operating on Thermal Neutrons: Énergoatomizdat, Moscow.

Hoover, W. G., \& Hoover, C. (2003). Links between microscopic and macroscopic fluid mechanics. Molecular Physics, 101(11), 1559-1573.

Kuchling, H. (1980). Physics. Veb Fachbuchverlag, Leipzig Loschmidt constant, (n.d.). from http://en.wikipedia.org/wiki/Loschmidt\%27s_number

Martel, L. M. V., . (2013). Magnesium-rich Basalts on Mercury, $\quad$ Retrieved from. from http://www.psrd.hawaii.edu/May13/Mercury-Mg-richcrust.html

Mohideen, U., \& Roy, A. (1998). Precision measurement of the Casimir force from 0.1 to $0.9 \mu \mathrm{m}$. Physical Review Letters, 81(21), 4549.

Mohr, P. J., \& Taylor, B. N. (2005). CODATA recommended values of the fundamental physical constants: 2002. Reviews of Modern Physics, 77(1), 1.
NASA. (2014). Neptune's magnetic environment, Retrieved from.http://voyager.jpl.nasa.gov/science/neptune_magnetic .html

NASA. ( 2011). What Is Jupiter? Retrieved from http://www.nasa.gov/audience/forstudents/58/features/what-is-jupiter-58.html\#.U1K5WlXwuSp

Nuclear Data Center.

Retrieved 46-Pd-106, from http://wwwndc.jaea.go.jp/cgibin/Tab80WWW.cgi?/data/JENDL/JENDL-4prc/intern/Pd106.intern

Perrin, J. (1923). Atoms. translated by DL Hammick. London: Constable.

Perrin, J. (2013). Brownian movement and molecular reality: Courier Dover Publications.

Russell, C. T. ( 1981). Planetary magnetism. Adv. Space Res., 1, pp. 257-263.

Russell, C. T., \& Luhmann, J. G. (1997). Mercury: Magnetic field and magnetosphere. Enciclopedia of Planetary Sciences, 476-478.

Ryder, L. (1985). Quantum field theory, 1985. Cambridge, Uk: Univ. Pr.

Salam, A., \& Sivaram, C. (1993). Strong gravity approach to QCD and confinement. Modern Physics Letters A, 8(04), 321-326.

Virgo, S. (1933). Loschmidt's number. Science Progress, 27, 634-649.

Zhang, T., Lu, Q., Baumjohann, W., Russell, C., Fedorov, A., Barabash, S., Nakamura, R. (2012). Magnetic reconnection in the near Venusian magnetotail. Science, 336(6081), 567570.

Zhussupov, M. (2011a,). Thermodynamic Criterions for Association of the Four Fundamental Interactions, Thermodynamics 2011 Conference, (p. 431), .

Zhussupov, M. (2011b). An Astronomical Criterion for Association of the Four Fundamental Interactions. Kepler Science Conference, (p. 155).

Zhussupov, M. (2011c, ). e-Chemist. Thermodynamics 2011 Conference, (p. 508).

Zhussupov, M. (2013). Feature of Thermodynamic Modelling on the Base of Unification of the Fundamental Interactions as an Alternative to Quantum Mechanics. Thermodynamics Conference 